\title{
STATIC AND SENSITIVITY ANALYSIS OF NONLOCAL NANOBEAMS SUBJECT TO LOAD AND MATERIAL UNCERTAINTIES BY CONVEX MODELING
}

\author{
IsAaC SFISO RADEBE \\ Department of Mechanical Engineering, Durban University of Technology, Durban, South Africa \\ e-mail:sfisor@dut.ac.za \\ SARP ADALI \\ Discipline of Mechanical Engineering, University of KwaZulu-Natal, Durban, South Africa \\ e-mail: adali@ukzn.ac.za
}

\begin{abstract}
At the nano-scale, loads acting on a nanobeam and its material properties are likely to be not known precisely, i.e., uncertain. In the present paper, the deflection of a nanobeam subject to load and material uncertainties is studied by convex modeling of the uncertainties. The level of uncertainty is taken to be bounded and the maximum deflection corresponding to the worst-case of loading or material properties is obtained, that is, the uncertainties are determined so as to maximize the deflection. The sensitivity of the deflection to the uncertainty in the material properties is also investigated. Numerical results are given relating the level of uncertainty to maximum deflection.
\end{abstract}

Keywords: nanobeams, load uncertainty, material uncertainty, convex modeling, sensitivity

\section{Introduction}

Mechanics of nanobeams has been studied extensively using continuum based models in an effort to quantify their behavior under static, buckling and dynamic loads. Several studies focused on continuum modeling and mechanics of nano and micro-sized beams and carbon nanotubes using various beam theories (see Wang and Shindo, 2006; Reddy, 2007; Reddy and Pang, 2008; Adali, 2008; Zhang et al., 2010; Di Paola et al., 2011; Muc, 2011; Adali, 2011; Hosseini-Ara et al., 2012; Thai, 2012; Thai and Vo, 2012; Eltaher et al., 2013). These studies employed Euler-Bernoulli and Timoshenko beam models coupled with the nonlocal elastic theory (see Eringen, 2002) to formulate the variational principles and the governing equations for nanobeams undergoing static bending, buckling and vibrations.

The bending behavior of nano-scale structures has been the subject of several studies and, in particular, nanobeams under static transverse loads were studied in (Wang and Shindo, 2006; Reddy, 2007; Reddy and Pang, 2008; Zhang et al., 2010; Di Paola et al., 2011; Thai, 2012; Thai and Vo, 2012; Eltaher et al., 2013; Challamel and Wang, 2008; Wang et al., 2008; Ansari and Sahmani, 2011; Fang et al., 2011; Roque et al., 2011; Li et al., 2012; Khajeansari et al., 2012). These studies took into account a number of effects such as shear deformation, surface stress, and elastic foundation but neglected the load and material uncertainties.

As such in the previous studies, the loading was taken as deterministic and the material properties were defined taking their average values. However, under operational conditions, the loads often have random characteristics making it difficult to predict their magnitude and distribution with accuracy. Similarly, it is usually difficult to determine the elastic constants of nano-sized beams with some certainty. The scatter in the geometric and material properties of 
carbon nanotubes is known and was discussed by Kalamkarov et al. (2006), Huang et al. (2006), Scarpa and Adhikari (2008), Lu and Zhong (2012) and Fereidoon et al. (2014).

The main trust of the present work is to study the bending of nanobeams in a nondeterministic setting by taking the load and material variations into account. Thus the maximum deflection of nanobeams is determined taking the transverse loading as non-deterministic and the material properties as uncertain. The problem analysis is conducted using convex modeling of uncertainties to determine the least favourable conditions to produce the highest deflection. Convex modeling has been used extensively in the past to deal with various engineering problems containing data uncertainties (see Adali et al., 1995a,b; Pantelidis and Ganzerli, 1998; Jiang et al., 2007; Kang and Luo, 2009; Hu and Qiu, 2010; Radebe and Adali, 2013). For further information, the reader is referred to the review articles by Wang et al. (2001) and the book by Ben-Haim and Elishakoff (1990).

In the present study, the effect of load and material uncertainties on the deflection of a nanobeam is studied based on the nonlocal Euler-Bernoulli beam theory. The previous work on the subject involves the study of the effect of material uncertainties on the buckling of a nonlocal plate by Radebe and Adali (2014). Load and material uncertainties are modeled as uncertainbut-bounded quantities. Explicit expressions are obtained for the least favorable deflection of a nanobeam for a given level of uncertainty. The sensitivity of the deflection to the level of uncertainty in material properties is also studied. Numerical results are given to investigate the effect of uncertainty on the deflection and on the sensitivity to material properties.

\section{Load uncertainty}

The nanobeam under consideration is subject to a combination of deterministic and uncertain transverse loads denoted by $p(x)$ and $\widetilde{q}(x)$, respectively, as well as a compressive axial load $N_{0}$ as shown in Fig. 1. The beam has a rectangular cross-section of dimensions $h \times b$, where $h$ is the height and $b$ is the width (Fig. 1).
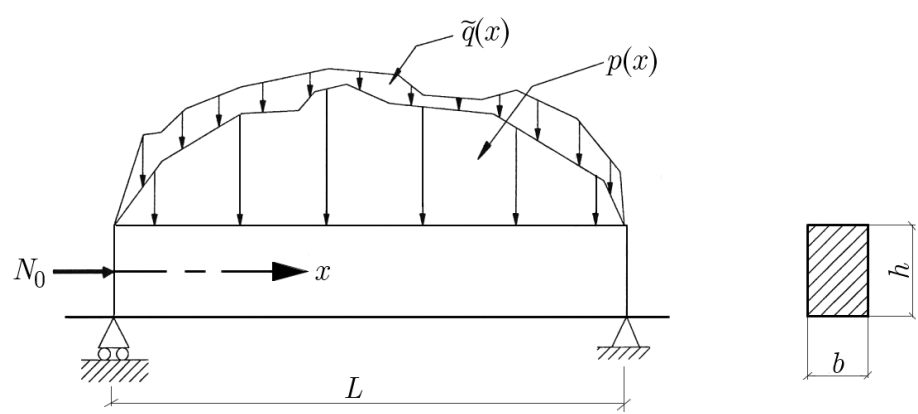

Fig. 1. Beam geometry and uncertain loading

The differential equation governing its deflection $w(x)$ based on the nonlocal elastic theory is given by (Reddy, 2007)

$$
E I w_{x x x x}+N_{0}\left(w_{x x}-\eta^{2} w_{x x x x}\right)=\left(p-\eta^{2} p_{x x}\right)+\left(\widetilde{q}-\eta^{2} \widetilde{q}_{x x}\right) \quad \text { for } \quad 0 \leqslant x \leqslant L
$$

where $E$ is Young's modulus, $I$ is the moment of inertia and $\eta$ is the small-scale parameter. The subscript $x$ denotes differentiation with respect to $x$. The compressive axial load $N_{0}$ satisfies the buckling constraint $N_{0}<N_{c r}$, where the buckling load $N_{c r}$ is given by (see Reddy, 2007)

$$
N_{c r}=\frac{\mu^{2} E I}{L^{2}+\mu^{2} \eta^{2}}
$$

with $\mu$ denoting a coefficient depending on the boundary conditions. 
The uncertain load $\widetilde{q}(x)$ acting on the beam is unknown, and only limited information is available on its coefficients. The information required on the uncertain load is that it should have a finite norm, i.e., it should satisfy the constraint

$$
\|\widetilde{q}(x)\|_{L_{2}}^{2}=\int_{0}^{L}[\widetilde{q}(x)]^{2} d x \leqslant \varepsilon^{2}
$$

where $0<\varepsilon<1$ is a given constant which determines the level of uncertainty, and the subscript $L_{2}$ denotes the $L_{2}$ norm. The solution to the deflection problem is obtained by expanding the deterministic and uncertain loads in terms of orthogonal functions $\psi_{n}(x)$ satisfying the boundary conditions, viz.

$$
p(x)=\sum_{n=1}^{\infty} p_{n} \psi_{n}(x) \quad \widetilde{q}(x)=\sum_{n=1}^{\infty} \widetilde{q}_{n} \psi_{n}(x)
$$

where the coefficients are given by

$$
p_{n}=\frac{1}{r} \int_{0}^{L} p(x) \psi_{n}(x) d x \quad \widetilde{q}_{n}=\frac{1}{r} \int_{0}^{L} \widetilde{q}(x) \psi_{n}(x) d x
$$

with $r$ given by

$$
r=\left\|\psi_{n}(x)\right\|_{L_{2}}^{2}=\int_{0}^{L}\left[\psi_{n}(x)\right]^{2} d x
$$

Here, the coefficients $p_{n}$ are known since the deterministic load $p(x)$ is given, however the coefficients $\widetilde{q}_{n}$ are unknown and have to be determined to maximize the deflection corresponding to the least favourable (worst-case) loading. The solution for the deflection function $w(x)$ is also expanded in terms of $\psi_{n}(x)$ and can be expressed as

$$
w(x)=\sum_{n=1}^{\infty} W_{n} \psi_{n}(x)
$$

The coefficients $W_{n}$ are computed by substituting Eq. (2.7) into differential equation (2.1). Next, the worst-case uncertain loading causing the highest deflection is obtained. From Eqs. (2.3) and (2.5), it follows that

$$
\sum_{n=1}^{N}\left(\widetilde{q}_{n}\right)^{2} \leqslant \frac{\varepsilon^{2}}{r}
$$

where $N$ is a large number. The highest load is obtained when $\sum_{n=1}^{N}\left(\widetilde{q}_{n}\right)^{2}=\varepsilon^{2} / r$, i.e., the inequality is taken as an equality. Thus the deflection $w(x ; \widetilde{q})$ is to be maximized with respect to the uncertain load subject to the constraint $\sum_{n=1}^{N}\left(\widetilde{q}_{n}\right)^{2}=\varepsilon^{2} / r$. For this purpose, the method of Lagrange multipliers is employed with the Lagrangian at a point $x=x_{0}$ given by

$$
L\left(x_{0} ; \widetilde{q}_{n}\right)=w\left(x_{0} ; \widetilde{q}\left(x_{0}\right)\right)+\lambda\left(\sum_{n=1}^{N}\left(\widetilde{q}_{n}\right)^{2}-\frac{\varepsilon^{2}}{r}\right)
$$

where $\lambda$ is a Lagrange multiplier and $0 \leqslant x_{0} \leqslant L$ is a point which has to be determined such that $w\left(x_{0} ; \widetilde{q}\left(x_{0}\right)\right)$ is maximum at $x=x_{0}$. The maximum of $L\left(x_{0} ; \widetilde{q}_{n}\right)$ with respect to $\widetilde{q}_{n}$ produces the least favourable uncertain load, viz.

$$
\max _{\widetilde{q}_{n}} L\left(x_{0} ; \widetilde{q}_{n}\right)
$$


which can be computed by setting its derivative with respect to $\widetilde{q}_{n}$ to zero, viz.

$$
\frac{\partial L\left(x_{0} ; \widetilde{q}_{n}\right)}{\partial \widetilde{q}_{n}}=0 \quad \text { for } \quad n=1,2, \ldots, N
$$

This computation gives the coefficient $\widetilde{q}_{n}$ at a point $x_{0}$ as

$$
\widetilde{q}_{n}\left(x_{0}\right)=-\frac{1}{2 \lambda} \frac{\partial w\left(x_{0} ; \widetilde{q}_{n}\left(x_{0}\right)\right)}{\partial \widetilde{q}_{n}}
$$

The point $x_{0}$ is an unknown and has to be determined to maximize the deflection.

\subsection{Simply supported beam}

The method of solution outlined above is now applied to a simply supported beam subject to the deterministic load $p(x)=p_{0}(x / L)^{3}$ and the uncertain load $\widetilde{q}(x)$. The simply supported boundary conditions for the nonlocal nanobeam are given by (Reddy, 2007)

$$
\begin{array}{ll}
w(0)=0 & \left(-E I+\eta^{2} N_{0}\right) w_{x x}(0)+\eta^{2} k_{0} b w(0)-\eta^{2} p(0)-\eta^{2} \widetilde{q}(0)=0 \\
w(L)=0 & \left(-E I+\eta^{2} N_{0}\right) w_{x x}(L)+\eta^{2} k_{0} b w(L)-\eta^{2} p(L)-\eta^{2} \widetilde{q}(L)=0
\end{array}
$$

The deterministic and uncertain loads are expanded in terms of the orthogonal functions $\psi_{n}(x)=\sin \alpha_{n} x$, where $\alpha_{n}=(n \pi) / L$. Thus

$$
p(x)=\sum_{n=1}^{N} p_{n} \sin \alpha_{n} x \quad \widetilde{q}(x)=\sum_{n=1}^{N} \widetilde{q}_{n} \sin \alpha_{n} x
$$

here the coefficients $p_{n}$ are given by

$$
p_{n}=(-1)^{n+1} \frac{2 p_{0}}{(n \pi)^{3}}\left(n^{2} \pi^{2}-6\right)
$$

The deflection $w(x)$ satisfying boundary conditions (2.13) can be obtained by expanding it in terms of $\sin \alpha_{n} x$ as

$$
w(x)=\sum_{n=1}^{N} W_{n} \sin \alpha_{n} x
$$

Substituting Eq. (2.16) into differential equation (2.1), the coefficients $W_{n}$ are computed as

$$
W_{n}=\frac{\left(1+\eta^{2} \alpha_{n}^{2}\right)\left(p_{n}+\widetilde{q}_{n}\right)}{E I \alpha_{n}^{4}-\left(1+\eta^{2} \alpha_{n}^{2}\right) \alpha_{n}^{2} N_{0}}
$$

the Lagrangian $L\left(x_{0} ; \widetilde{q}_{n}\right)$ given by Eq. (2.9) becomes

$$
L\left(x_{0} ; \widetilde{q}_{n}\right)=\sum_{n=1}^{N} \frac{\left(1+\eta^{2} \alpha_{n}^{2}\right)\left(p_{n}+\widetilde{q}_{n}\right)}{E I \alpha_{n}^{4}-\left(1+\eta^{2} \alpha_{n}^{2}\right) \alpha_{n}^{2} N_{0}} \sin \alpha_{n} x_{0}+\lambda\left(\sum_{n=1}^{N}\left(\widetilde{q}_{n}\right)^{2}-\frac{2 \varepsilon^{2}}{L}\right)
$$

The coefficients $\widetilde{q}_{n}\left(x_{0}\right)$ are computed from Eqs. (2.11) and (2.18) as

$$
\widetilde{q}_{n}\left(x_{0}\right)=-\frac{1}{2 \lambda} \frac{A_{n}\left(x_{0}\right)}{B_{n}}
$$

where

$$
A_{n}\left(x_{0}\right)=\left(1+\eta^{2} \alpha_{n}^{2}\right) \sin \alpha_{n} x_{0} \quad B_{n}=E I \alpha_{n}^{4}-\left(1+\eta^{2} \alpha_{n}^{2}\right) \alpha_{n}^{2} N_{0}
$$


Noting that the worst case loading is given by

$$
\sum_{n=1}^{N}\left(\widetilde{q}_{n}\right)^{2}=\frac{2 \varepsilon^{2}}{L}
$$

we can compute the Lagrange multiplier from Eqs. (2.19) and (2.21) as

$$
\lambda= \pm \frac{\sqrt{L}}{\sqrt{8} \varepsilon} \sqrt{\sum_{n=1}^{N} \frac{A_{n}^{2}\left(x_{0}\right)}{B_{n}^{2}}}
$$

where the plus and minus signs correspond to the least and most favourable loading cases. The coefficients $\widetilde{q}_{n}$ can be computed by inserting the Lagrange multiplier (2.22) into Eq. (2.19). This computation gives

$$
\widetilde{q}_{n}\left(x_{0}\right)=\mp \frac{\sqrt{2} \varepsilon}{\sqrt{L}}\left(\sqrt{\sum_{n=1}^{N} \frac{A_{n}^{2}\left(x_{0}\right)}{B_{n}^{2}}}\right)^{-1} \frac{A_{n}\left(x_{0}\right)}{B_{n}}
$$

The uncertain load producing the maximum deflection is given by Eq. (2.14) with the coefficients given by Eq. (2.23).

\section{Material uncertainty}

\subsection{Uncertain constants}

Next, the effect of uncertainty in material properties on the deflection is investigated. Young's modulus $\widetilde{E}$ and the small scale parameter $\widetilde{\eta}$ are taken as uncertain material parameters, and they are defined as

$$
\widetilde{E}=E_{0}\left(1+\delta_{1}\right) \quad \widetilde{\eta}=\eta_{0}\left(1+\delta_{2}\right)
$$

where $E_{0}$ and $\eta_{0}$ are the nominal (deterministic) values, and $\delta_{1}$ and $\delta_{2}$ are margins of error (uncertainty) to be determined to maximize the deflection. The unknown constants $\delta_{1}$ and $\delta_{2}$ are required to lie in an ellipse and satisfy the inequality $\sum_{i=1}^{2} \delta_{i}^{2} \leqslant \gamma^{2}$ which corresponds to inequality (2.3) of the uncertain loading case. The least favourable solution is given when the constants lie on the boundary of the ellipse, i.e., they satisfy the equality constraint

$$
\sum_{i=1}^{2} \delta_{i}^{2}=\gamma^{2}
$$

The material uncertainty is studied for a simply supported nanobeam under a sinusoidal load $p(x)=p_{1} \sin (\pi x / L)$. For this case, the maximum deflection occurs at the mid-point and is given by

$$
w\left(\frac{L}{2} ; \widetilde{E}, \widetilde{\eta}\right)=\frac{\left(1+\alpha_{1}^{2} \widetilde{\eta}^{2}\right) p_{1}}{\alpha_{1}^{4} I \widetilde{E}-\left(1+\alpha_{1}^{2} \widetilde{\eta}^{2}\right) \alpha_{1}^{2} N_{0}}
$$

where $\alpha_{1}=\pi / L$. Substituting Eq. (3.1) into Eq. (3.3), we obtain

$$
w\left(\frac{L}{2} ; \widetilde{E}, \widetilde{\eta}\right)=\frac{\left[1+\alpha_{1}^{2} \eta_{0}^{2}\left(1+\delta_{2}\right)^{2}\right] p_{1}}{\alpha_{1}^{4} I E_{0}\left(1+\delta_{1}\right)-\left[1+\alpha_{1}^{2} \eta_{0}^{2}\left(1+\delta_{2}\right)^{2}\right] \alpha_{1}^{2} N_{0}}
$$


which can be linearized leading to the expression

$$
w\left(\frac{L}{2} ; \widetilde{E}, \widetilde{\eta}\right)=c_{0}+c_{1} \delta_{1}+c_{2} \delta_{2}
$$

where

$$
\begin{array}{ll}
c_{0}=\frac{1+\alpha_{1}^{2} \eta_{0}^{2}}{\alpha_{1}^{4} I E_{0}-\left(1+\alpha_{1}^{2} \eta_{0}^{2}\right) \alpha_{1}^{2} N_{0}} p_{1} \quad c_{1}=-\frac{I E_{0}\left(1+\alpha_{1}^{2} \eta_{0}^{2}\right)}{\left[\alpha_{1}^{2} I E_{0}-\left(1+\alpha_{1}^{2} \eta_{0}^{2}\right) N_{0}\right]^{2}} p_{1} \\
c_{2}=\frac{2 \alpha_{1}^{2} I E_{0} \eta_{0}^{2}}{\left[\alpha_{1}^{2} I E_{0}-\left(1+\alpha_{1}^{2} \eta_{0}^{2}\right) N_{0}\right]^{2}} p_{1}
\end{array}
$$

To derive expression (3.5), the relation

$$
(1 \pm \delta)^{c} \cong(1 \mp c \delta)+O\left(\delta^{2}\right)
$$

is employed, where the superscript $c$ can take positive or negative values and $|\delta| \ll 1$. The Lagrangian $L\left(\delta_{1}, \delta_{2}\right)$ to compute the maximum deflection subject to constraint (3.2) is given by

$$
L\left(\delta_{1}, \delta_{2}\right)=c_{0}+c_{1} \delta_{1}+c_{2} \delta_{2}+\lambda\left(\sum_{n=1}^{2} \delta_{i}^{2}-\gamma^{2}\right)
$$

The constants $\delta_{i}$ are computed from Eq. (3.8) as

$$
\delta_{i}=-\frac{c_{i}}{2 \lambda}
$$

The Lagrange multiplier $\lambda$ can be computed from Eqs. (3.2) and (3.9) as

$$
\lambda= \pm \frac{1}{2 \gamma} \sqrt{\sum_{i=1}^{2} c_{i}^{2}}
$$

where the plus and minus signs correspond to least and most favourable cases. The coefficients $\delta_{i}$ can be computed by inserting Lagrange multiplier (3.10) into Eq. (3.9). This computation gives

$$
\delta_{i}=\mp \gamma \frac{1}{\sqrt{c_{1}^{2}+c_{2}^{2}}} c_{i}
$$

The values of $\delta_{i}$ given by Eq. (3.11) are substituted into Eq. (3.5) to compute the mid-point deflection $w(L / 2 ; \widetilde{E}, \widetilde{\eta})$ subject to material uncertainty.

\subsection{Sensitivity analysis}

The sensitivity of the deflection to the level of uncertainty in material data can be studied by sensitivity analysis. In general, the deflection shows different sensitivities to the material parameters $\widetilde{E}$ and $\widetilde{\eta}$, and this can be investigated by defining relative sensitivity indices $S_{K}\left(\delta_{i}\right)$ given by

$$
S_{K}\left(\delta_{i}\right)=\left|\frac{\partial w(L / 2 ; \widetilde{E}, \widetilde{\eta})}{\partial \delta_{i}}\right| \frac{\left|\delta_{i}\right|}{w\left(L / 2 ; E_{0}, \eta_{0}\right)}
$$

which is normalized with respect to the deterministic mid-point deflection $w\left(L / 2 ; E_{0}, \eta_{0}\right)$. In Eq. (3.12), the sensitivity $S_{E}\left(\gamma_{1}\right)$ denotes the relative sensitivity of the mid-point deflection with respect to uncertainty in $\widetilde{E}$, and $S_{\eta}\left(\delta_{2}\right)$ with respect to uncertainty in $\widetilde{\eta}$ so that the subscript $K$ stands for the respective material property. The sensitivities $S_{K}\left(\delta_{i}\right)$ can be computed from Eqs. (3.5) and (3.12) as

$$
S_{K}\left(\delta_{i}\right)=\frac{\left|c_{i} \delta_{i}\right|}{c_{0}}
$$

noting that $w\left(L / 2 ; E_{0}, \eta_{0}\right)=c_{0}$ where the values of $c_{i}$ are given by Eqs. (3.6). 


\section{Numerical results}

The effect of uncertain loads and material properties on the deflection is studied in the present section. The cross-section of the nanobeam is taken as square, and the height and the length of the nanobeam are specified as $b=h=1 \mathrm{~nm}$ and $L=10 \mathrm{~nm}$. The material properties are specified as $E=1000 \mathrm{GPa}, 0 \leqslant \eta \leqslant 2 \mathrm{~nm}$.

\subsection{Load uncertainty}

$L_{2}$ norms of the uncertain and the deterministic loads can be related as

$$
\|\widetilde{q}(x)\|_{L_{2}}^{2}=\varepsilon^{2}=R_{0}^{2}\|p(x)\|_{L_{2}}^{2}
$$

where $R_{0}$ is a proportionality constant and determines the degree of uncertainty relative to the deterministic load with $R_{0}=0$ corresponding to no uncertainty, i.e., the deterministic case. For the present case $\|p(x)\|_{L_{2}}^{2}=p_{0}^{2} L / 7$, hence

$$
\varepsilon=\sqrt{\frac{L}{7}} p_{0} R_{0}
$$

In the calculations, the load coefficient $p_{0}$ is taken as $p_{0}=1 \mathrm{~N} / \mathrm{m}$. Figure 2 shows curves of the deflection vs. $x$-axis for various uncertainty levels $R_{0}$ with $\eta=2 \mathrm{~nm}$ and $N_{0}=0$. In Fig. 2 and in the subsequent figures, the curves are obtained by setting $x_{0}=x$ in equation (2.7), and consequently at every point $x$ the deflection is the least favourable deflection.

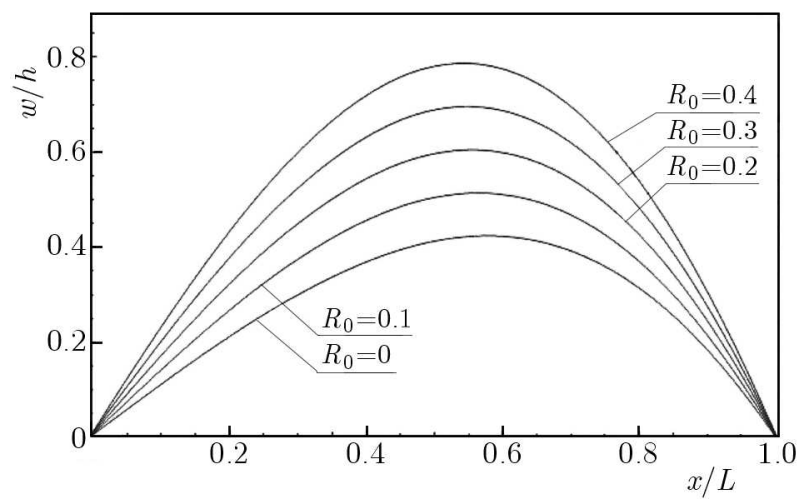

Fig. 2. Deflection curves vs. $x$-axis for various uncertainty levels with $\eta_{0}=2 \mathrm{~nm}$ and $N_{0}=0$

Figure 2 shows that, compared to the deterministic case corresponding to $R_{0}=0$, the deflection increases as the level of load uncertainty increases. The corresponding results for a beam subject to a compressive axial load of $N_{0}=0.5 N_{c r}$ are given in Fig. 3 which shows the effect of compressive axial load on the uncertain deflection. For a simply supported beam, the coefficient $\mu=\pi$ in (2.2) for $N_{c r}$.

The effect of the small scale parameter $\eta_{0}$ on the deflection of the nanobeam subject to an uncertain load with $R_{0}=0.3$ is shown in Fig. 4. It is observed that both the small-scale parameter $\eta_{0}$ and the level of deflection are factors in the increasing of the mid-point deflection. Next, the combined effect of the small-scale parameter and the axial load on the maximum deflection is studied in Fig. 5 which shows the contour plots of the maximum deflection with respect to $0 \leqslant N_{0} \leqslant 0.6 N_{c r}$ ( $x$-axis) and $0 \leqslant \eta_{0} \leqslant 2 \mathrm{~nm}$ (y-axis) for uncertainty levels $0 \leqslant R_{0} \leqslant 0.3$. The maximum deflection of the beam is computed by

$$
\max _{0 \leqslant x \leqslant L} w(x)=\max _{0 \leqslant x \leqslant L}\left(\sum_{n=1}^{N} W_{n} \sin \alpha_{n} x\right)
$$




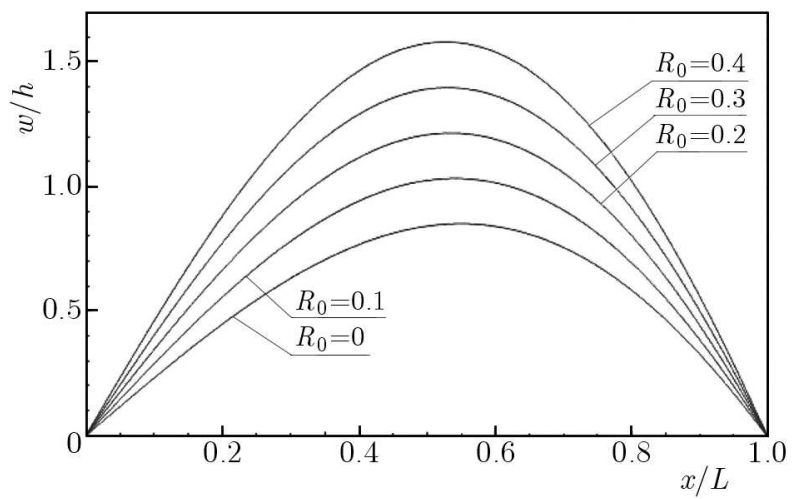

Fig. 3. Deflection curves vs. $x$-axis for various uncertainty levels with $\eta_{0}=2 \mathrm{~nm}$ and $N_{0}=0.5 N_{c r}$

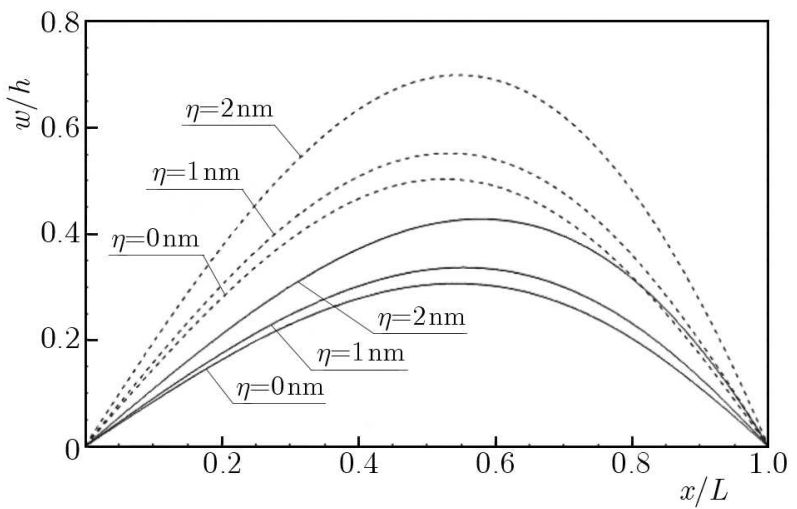

Fig. 4. Deflection curves vs. $x$-axis for the deterministic case (full lines) and for an uncertainty level of $R_{0}=0.3$ (dotted line) with $\eta_{0}=0,1,2 \mathrm{~nm}$ and $N_{0}=0$

using a minimization routine in Mathematica. Figure 5 shows that an increase in the parameters $\eta_{0}$ or $N_{0}$ as well as in the level of load uncertainty leads to higher deflection.

(a)

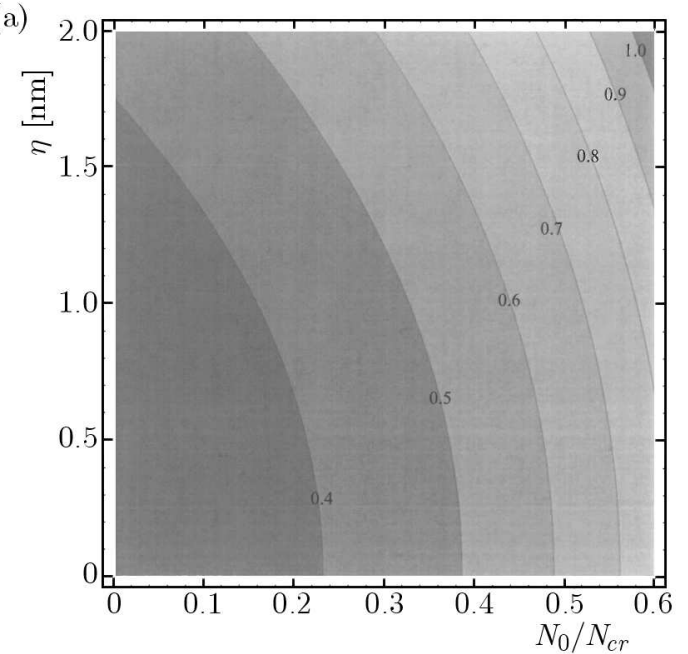

(b)

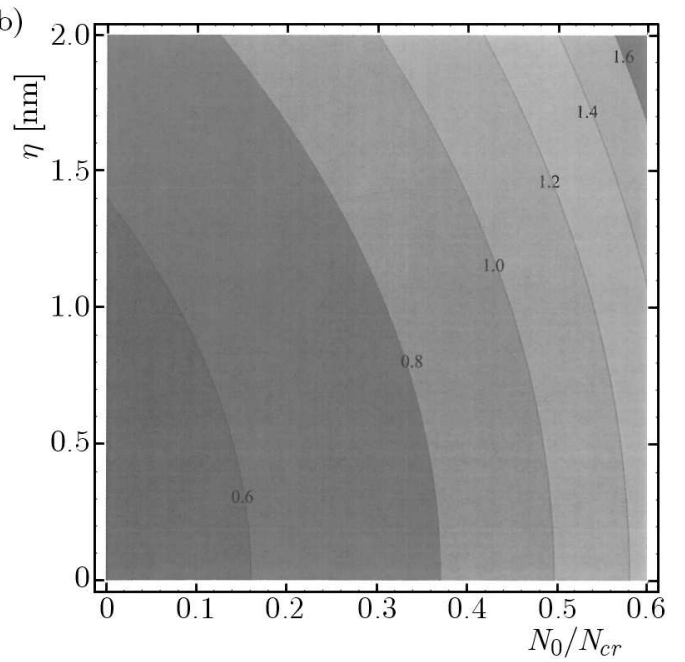

Fig. 5. Contour plots of the maximum deflection with respect to $N_{0}$ ( $x$-axis) and $\eta$ ( $y$-axis) for: (a) $R_{0}=0$, (b) $R_{0}=0.3$

\subsection{Material uncertainty}

Next, numerical results are given for the problem studied in Section 3 for a square nanobeam of $b=h=1 \mathrm{~nm}$ and length $L=10 \mathrm{~nm}$ with $p_{1}=0.1 \mathrm{~N} / \mathrm{m}$ and $N_{0}=0$. The nominal 
(deterministic) value of Young's modulus is taken as $E_{0}=1000 \mathrm{GPa}$. The results in the following figures are obtained by employing exact expression (3.4) for the mid-point deflection of the nanobeam. In the figures, the mid-point deflection is normalized by the height $h$ by defining $w_{0}=w(L / 2 ; \widetilde{E}, \widetilde{\eta}) / h$.

Figure 6 shows the curves of mid-point deflection $w_{0}$ plotted against the uncertainty level $\gamma$ for various values of the uncertain small-scale parameter $\eta_{0}$. It is observed that the maximum deflection increases with increasing material uncertainty and the increase is given by a nonlinear curve. The effect of the small-scale parameter $\eta_{0}$ on the mid-point deflection is shown in Fig. 7 . It is observed that the effect of uncertainty becomes more pronounced at higher values of the small-scale parameter.

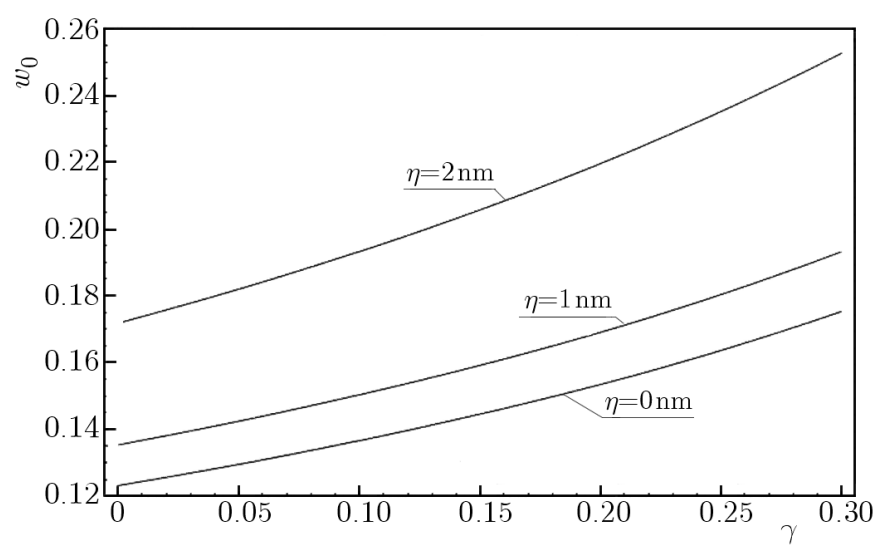

Fig. 6. Mid-point deflection vs. the uncertainty parameter $\gamma$ for various values of $\eta_{0}$

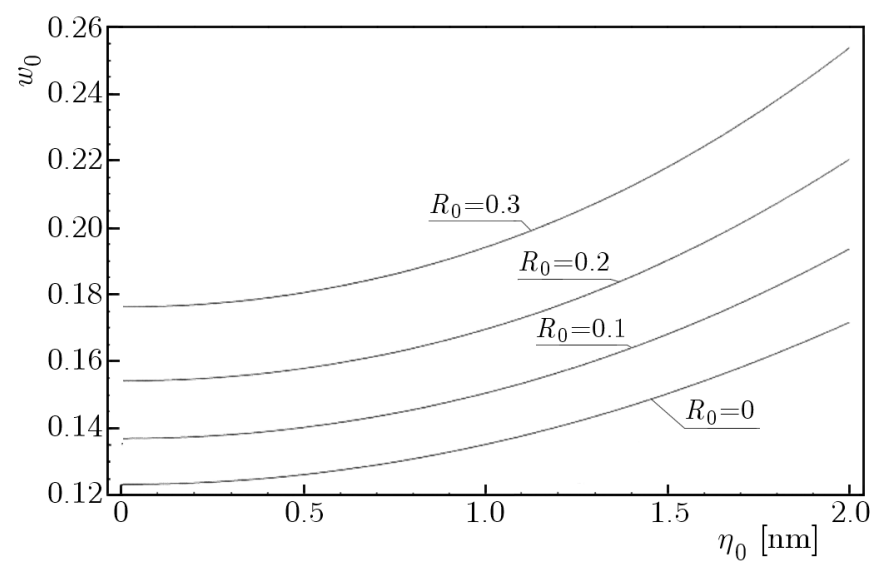

Fig. 7. Mid-point deflection vs. the small-scale parameter for various levels of uncertainty

Next, the sensitivity of the deflection to material properties is studied in Fig. 8 which shows the contour plots of the mid-point deflection $w_{0}$ with respect to the level of uncertainty and the small-scale parameter. It is observed that the sensitivity of the deflection with respect to Young's modulus is about 5 times more than the sensitivity to the small-scale parameter. Moreover, the sensitivity with respect to Young's modulus is not affected much with respect to the small-scale parameter, but the sensitivity with respect to the small-scale parameter increases with growing $\eta_{0}$. 
(a)

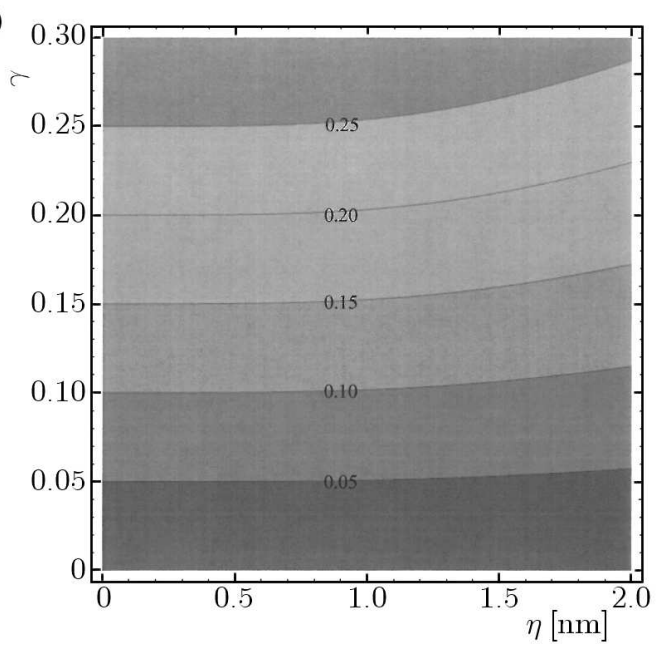

(b)

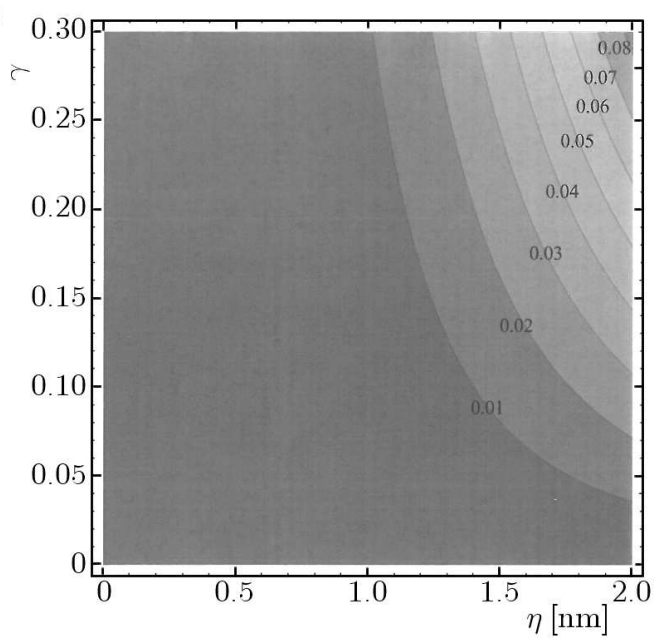

Fig. 8. Contour plots of sensitivities with respect to the level of uncertainties and small-scale parameter: (a) $S_{E}$, (b) $S_{\eta}$

\section{Conclusions}

Non-probabilistic analysis of the uncertainties which can arise in transverse loads and in material properties of nanobeams is given using convex modeling. The variations in uncertain quantities are taken as uncertain-but-bounded by imposing a constraint on the $L_{2}$ norm of the uncertainties. The nanobeam is modeled as a nonlocal Euler-Bernoulli beam and the effect of axial compression is taken into account. The uncertain load is approximated by a Fourier series expression and the coefficients of the series are determined to obtain the worst-case uncertain loading. Closed form solutions of the problems are given, and the theory is illustrated for simply supported boundary conditions. It is observed that the increasing uncertainty as manifested by increasing the $L_{2}$ norm of the uncertain load leads to higher deflections. The effect of uncertainties in Young's modulus and the small-scale parameter is also studied and a sensitivity index is proposed to assess the sensitivity of the deflection to these parameters. Numerical results are given to observe the effect of various problem parameters on the deflection. The present study complements the studies in the literature on the static deflection of nanobeams which have taken the loads acting on the nanobeams and its properties as deterministic neglecting the uncertainties which can occur under operational conditions.

\section{Acknowledgements}

The research reported in this paper was supported by research grants from the University of KwaZuluNatal (UKZN) and from National Research Foundation (NRF) of South Africa. The author gratefully acknowledges the supports provided by UKZN and NRF.

\section{References}

1. AdALi S., 2008, Variational principles for multi-walled carbon nanotubes undergoing buckling based on nonlocal elasticity theory, Physics Letters A, 372, 5701-5705

2. ADALI S., 2011, Variational principles for vibrating carbon nanotubes modeled as cylindrical shells based on strain gradient nonlocal theory, Journal of Computational and Theoretical Nanoscience, 8,1954-1962

3. Adali S., Richter A., Verijenko V.E., 1995a, Minimum weight design of symmetric angle-ply laminates under multiple uncertain loads, Structural Optimization, 9, 89-95 
4. Adali S., Richter A., Verijenko V.E., 1995b, Non-probabilistic modelling and design of sandwich plates subject to uncertain loads and initial deflections, International Journal of Engineering Science, 33, 855-866

5. Ansari R., Sahmani S., 2011, Bending behavior and buckling of nanobeams including surface stress effects corresponding to different beam theories, International Journal of Engineering Science, 49, 1244-1255

6. Ben-Haim Y., Elishakoff I., 1990, Convex Models of Uncertainty in Applied Mechanics, Elsevier Science Publishers, Amsterdam, The Netherlands.

7. Challamel N., Wang C.M., 2008, Small length scale effect in non-local cantilever beam: paradox solved, Nanotechnology, 19, 345703

8. Di Paola M., Failla G., Sofi A., Zingales M., 2011, A mechanically based approach to non-local beam theories, International Journal of Mechanical Science, 53, 676-687

9. Eltaher M.A., Emam S.A., Mahmoud F.F., 2013, Static and stability analysis of nonlocal functionally graded nanobeams, Composite Structures, 96, 82-88

10. Eringen A.C., 2002, Nonlocal Continuum Field Theories, Springer, New York

11. Fang C., Kumar A., Mukherjee S., 2011, A finite element analysis of single-walled carbon nanotube deformation, ASME Journal of Applied Mechanics, 78, 034502-1-034502-7

12. Fereidoon A., Rajabpour M., Hemmatian H., 2014, Elastic moduli of carbon nanotubes with new geometry based on FEM, Journal of Theoretical and Applied Mechanics, 52, to appear

13. Hosseini-Ara R., Mirdamadi H.R., Khademyzadeh H., 2012, Buckling analysis of short carbon nanotubes based on a novel Timoshenko beam model, Journal of Theoretical and Applied Mechanics, 50, 975-986

14. Hu J., QIU Z., 2010, Non-probabilistic convex models and interval analysis method for dynamic response of a beam with bounded uncertainty, Applied Mathematical Modelling, 34, 725-734

15. Jiang C., Han X., LiU G.R., 2007, Optimization of structures with uncertain constraints based on convex model and satisfaction degree of interval, Computer Methods in Applied Mechanics and Engineering, 196, 4791-4800

16. Kalamkarov A.L., Georgiades A.V., Rokkam S.K., Veedu V.P., Ghasemi-Nejhad M.N., 2006, Analytical and numerical techniques to predict carbon nanotubes properties, International Journal of Solids and Structures, 43, 6832-6854

17. KANG Z., LuO Y., 2009, Non-probabilistic reliability-based topology optimization of geometrically nonlinear structures using convex models, Computer Methods in Applied Mechanics and Engineering, 198, 3228-3238

18. Khajeansari A., Baradaran G.H., Yvonnet J., 2012, An explicit solution for bending of nanowires lying on Winkler-Pasternak elastic substrate medium based on the Euler-Bernoulli beam theory, International Journal of Engineering Science, 52,115-128

19. Li X.-F., WAng B.-L., TANG G.-J., LeE K.Y., 2012, Size effect in transverse mechanical behaviour of one-dimensional nanostructures, Physica E, Low-dimensional Systems and Nanostructures, 44, 207-214

20. Lu X., Zhong H., 2012, Mechanical property evaluation of single-walled carbon nanotubes by finite element modeling, Composites Part B: Engineering, 43, 1902-1913

21. Muc A., 2011, Modelling of carbon nanotubes behaviour with the use of a thin shell theory, Journal of Theoretical and Applied Mechanics, 49, 531-540

22. Pantelidis C.P., Ganzerli S., 1998, Design of trusses under uncertain loads using convex models, ASCE Journal of Structural Engineering, 124, 318-329

23. Radebe I.S., Adali S., 2013, Minimum weight design of beams against failure under uncertain loading by convex analysis, Journal of Mechanical Science and Technology, 27, 2071-2078 
24. Radebe I.S., AdAli S., 2014, Buckling and sensitivity analysis of nonlocal orthotropic nanoplates with uncertain material properties, Composites Part B: Engineering, 56, 840-846

25. REDDy J.N., 2007, Nonlocal theories for bending, buckling and vibration of beams, International Journal of Engineering Science, 45, 288-307

26. Reddy J.N., PAng S.D., 2008, Nonlocal continuum theories of beams for the analysis of carbon nanotubes, Journal of Applied Physics, 103, 023511

27. Roque C.M.C., Ferreira A.J.M., Reddy J.N., 2011, Analysis of Timoshenko nanobeams with a nonlocal formulation and meshless method, International Journal of Engineering Science, 49, 976-984

28. Scarpa F., Adhikari S., 2008, Uncertainty modeling of carbon nanotube terahertz oscillators, Journal of Non-Crystalline Solids, 354, 4151-4156

29. Thai H.-T., 2012, A nonlocal beam theory for bending, buckling, and vibration of nanobeams, International Journal of Engineering Science, 52, 56-64

30. Thai H.-T., Vo T.P., 2012, A nonlocal sinusoidal shear deformation beam theory with application to bending, buckling, and vibration of nanobeams, International Journal of Engineering Science, 54, 58-66

31. Wang C.M., Kitipornchai S., Lim C.W., Eisenberger M., 2008, Beam bending solutions based on nonlocal Timoshenko beam theory, ASCE Journal of Engineering Mechanics, 134, 475-481

32. WAng Q., Shindo Y., 2006, Nonlocal continuum models for carbon nanotubes subjected to static loading, Journal of Mechanics of Materials and Structures, 1, 663-680

33. Wang X., Wang L., Elishakoff I., Qiu Z., 2011, Probability and convexity concepts are not antagonistic, Acta Mechanica, 219, 45-64

34. Zhang Y.Y., Wang C.M., Challamel N., 2010, Bending, buckling, and vibration of micro/nanobeams by hybrid nonlocal beam model, ASCE Journal of Engineering Mechanics, 136, $562-574$ 